\title{
Characterization of host microRNAs that respond to DNA virus infection in a crustacean
}

\author{
Tianzhi Huang, Dandan Xu and Xiaobo Zhang*
}

\begin{abstract}
Background: MicroRNAs (miRNAs) are key posttranscriptional regulators of gene expression that are implicated in many processes of eukaryotic cells. It is known that the expression profiles of host miRNAs can be reshaped by viruses. However, a systematic investigation of marine invertebrate miRNAs that respond to virus infection has not yet been performed.

Results: In this study, the shrimp Marsupenaeus japonicus was challenged by white spot syndrome virus (WSSV). Small RNA sequencing of WSSV-infected shrimp at different time post-infection $(0,6,24$ and $48 \mathrm{~h})$ identified 63 host miRNAs, 48 of which were conserved in other animals, representing 43 distinct families. Of the identified host miRNAs, 31 were differentially expressed in response to virus infection, of which 25 were up-regulated and six down-regulated. The results were confirmed by northern blots. The TargetScan and miRanda algorithms showed that most target genes of the differentially expressed miRNAs were related to immune responses. Gene ontology analysis revealed that immune signaling pathways were mediated by these miRNAs. Evolutionary analysis showed that three of them, miR-1, miR-7 and miR-34, are highly conserved in shrimp, fruit fly and humans and function in the similar pathways.

Conclusions: Our study provides the first large-scale characterization of marine invertebrate miRNAs that respond to virus infection. This will help to reveal the molecular events involved in virus-host interactions mediated by miRNAs and their evolution in animals.
\end{abstract}

Keywords: Invertebrate miRNAs, WSSV, Sequencing, EST assembly, Virus-host interaction, GO analysis, Evolution

\section{Background}

MicroRNAs (miRNAs) are a large class of small noncoding RNAs that are found in diverse eukaryotic organisms. They range in size from 18 to 26 nucleotides and are cut sequentially from the stem regions of long hairpin transcripts by two RNase III proteins, Drosha and Dicer [1-3]. The mature miRNA strand is liberated from the miRNA:miRNA* duplex and incorporated into the RNAinduced silencing complex, where it controls the expression of cognate mRNA through degradation or translation repression [4-8]. It is known that miRNAs have important roles in many eukaryotic cellular pathways, including developmental timing, cell differentiation and proliferation, apoptosis, energy metabolism, cancer and immune

\footnotetext{
* Correspondence: zxb0812@zju.edu.cn

Key Laboratory of Conservation Biology for Endangered Wildlife of Ministry of Education, Key Laboratory of Animal Virology of Ministry of Agriculture and College of Life Sciences, Zhejiang University, Hangzhou 310058, People's College of Life Scienc
Republic of China
}

defense [1,3,9-13]. Host miRNAs are believed to be key regulators of virus-host interactions [14-16]. To date, however, information about the pathways mediated by host miRNAs or their evolution is limited.

It has been reported that infections of some mammalian viruses can alter the host miRNA expression profiles, and the expression patterns of some host miRNAs change markedly over the time course of viral infection $[15,16]$. These changes reflect that the host miRNAs may have important roles in the virus-host interactions. These miRNAs may be involved in the host immunity to the virus invasion, or in virus infection to create favorable intracellular environments for virus replication. A systematic investigation of marine invertebrate miRNAs whose expression is altered in response to virus infection has not yet been performed [17]. Invertebrates, which do not possess a lymphocyte-based adaptive immune system, rely entirely on innate immunity. 
Since the first miRNAs, lin-4 and let-7, were identified in Caenorhabditis elegans as potential regulators of animal development [18,19], 15,172 miRNAs have been discovered from various organisms (miRNA Registry, Release 17.0, April 2011), including mammals, plants, insects, nematodes and viruses. These miRNAs have been identified through computational or experimental approaches [20]. Many miRNAs are conserved among related species, suggesting that their functions may be evolutionarily conserved [1,21-23]. Using phylogenetic conservation and the criterion of a precursor hairpin structure (a characteristic hairpin structure with small internal loops, with the mature miRNA embedded in the stem of the hairpin), various computer programs have been developed to predict miRNAs, such as TargetScan [24], miRanda [25], MiRAlign [26] and Srnaloop [27]. However, the computational approaches are limited to organisms whose whole genome sequences are available. Recently, the high-throughput sequencing approach has successfully been used to identify miRNAs from various organisms [28-30]. Although this approach may omit the miRNAs with low abundance, it remains the approach of choice for identification of miRNAs in organisms whose whole genome sequences are unavailable [31]. Despite the large number of miRNAs that have been deposited in the miRBase database, this database is likely to be far from saturated as abundant miRNAs are still undiscovered from unexploited organisms. To date, identifications of miRNAs are limited to non-marine species, and very little information is available about the miRNAs of marine organisms.

In this study, the shrimp miRNAs involved in virus infection were investigated. Shrimps are one of the most important groups of species in marine aquaculture. In the past few decades, worldwide shrimp culture has been threatened by viral diseases, especially that by the white spot syndrome virus (WSSV) [32]. Owing to the lack of a true adaptive immune response system like that of vertebrates, invertebrates rely completely on the innate immune system to resist virus invasion. The miRNAs of invertebrates in general and marine invertebrates in particular, in response to virus infection, remain to be studied. In the present study, the miRNAs of WSSV-challenged shrimp ( $M$. japonicus) were characterized. The results showed that 31 shrimp miRNAs defended against virus infection by regulating immune pathways. Some miRNAs were highly conserved in shrimp, fruit fly and humans and function in the similar pathways. Our study provides clues to the molecular events mediated by host miRNAs in host-virus interactions.

\section{Results}

Sequence analysis of shrimp miRNAs in response to WSSV infection

To get an overview of the host miRNAs expressed in response to virus infection, the small RNAs of WSSV- infected shrimp at various times after infection were sequenced. After removal of mRNA, rRNA, tRNA, snRNA and snoRNA sequences, high-throughput sequencing generated a total of 35,588,792 raw sequences, of which $25,009,711$ reads were mappable to miRBase 15.0 or to the shrimp GenBank expressed sequence tag (EST) database (Additional file 1). However, no sequence mapped to the WSSV genome sequence. The analyses showed that the majority of the non-redundant sequences were 20-24 nucleotides (nt) in length, which is typical for products processed by the enzyme Dicer (Figure 1). Direct sequencing also revealed that the sequences at the 3 ' ends of the miRNAs ( $52.7 \%$ of total reads) appeared to be more heterogeneous than those at the $5^{\prime}$ ends (18.9\% of total reads), suggesting that the $5^{\prime}$ ends of miRNAs have key roles in target recognition, such as roles as seed sequences.

To characterize the shrimp miRNA homologs, the miRNA sequences were compared using BLAST with miRBase 15.0 with an E-value similarity cutoff of $1 \mathrm{e}-10$. This revealed that 48 miRNAs [GenBank: JQ706251- JQ706298] were mapped to other known arthropod miRNAs and premiRNAs in miRBase 15.0. But the mapped pre-miRNAs could not be mapped to shrimp ESTs. Therefore, these sequences were identified as conserved miRNAs of shrimp and could be classified into 43 distinct families (Table 1 and Additional file 2). The remaining miRNAs with no homolog were compared using BLASTN with the shrimp EST database, allowing one or two mismatches between each pair of sequences. Hairpin structures were predicted in the mapped ESTs using the Mfold program. According to this criterion, a total of 15 miRNAs [GenBank: JQ706299-JQ706313] with no homolog in other animals were identified (Table 1 and Additional file 3), which might be specific to shrimp or not yet discovered

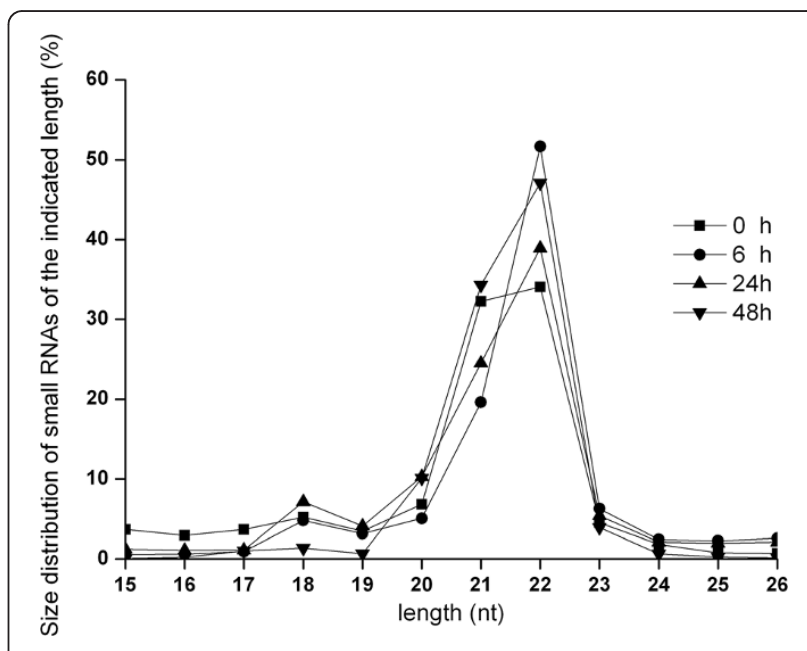

Figure 1 Size distribution of small RNAs found by sequencing. The symbols represented the time post- infection in hours. 
Table 1 Shrimp miRNAs up-regulated or down-regulated in response to WSSV infection at different time post-infection

\begin{tabular}{lll}
\hline Time post-infection & miRNAs conserved in animals & miRNAs with no homologue \\
\hline $\mathbf{0} \mathbf{h}$ & miR-1, miR-7, miR-9, miR-10a, miR-10*, miR-33, miR-34 miR-71, miR-79, miR-92a, & miR-S1, miR-S2 miR-S3, miR-S5 miR-S6, \\
& miR-92b, miR-100, miR-133 miR-184, miR-252, miR-71*, miR-let7, miR-2a, miR-2b & miR-S10 miR-S12, miR-S15 \\
& miR-2c, miR-8, miR-12, miR-87, miR-190, miR-193 miR-263a, miR-275, miR-276, & \\
& miR-276b, miR-279, miR-281 miR-282, miR-305, miR-315, miR-317, miR-750, & \\
& miR-965 miR-993, miR-1000, miR-276a*, miR-281-2*, miR-8*
\end{tabular}
miR-92b, miR-100, miR-133 miR-184, miR-252, miR-71*, miR-let7, miR-2a, miR-2b miR-S2 miR-2c, miR-8, miR-12, miR-87, miR-190, miR-193 miR-263a, miR-275, miR-276, miR-276b, miR-279, miR-281 miR-282, miR-305, miR-315, miR-317, miR-750, miR-965 miR-993, miR-1000, miR-276a*, miR-281-2*, miR-8* miR-252b, miR-278, miR-981, miR-bantam, miR-2001 miR-92b, miR-100, miR-133 miR-184, miR-252, miR-71*, miR-let7, miR-2a, miR-2b miR-2c, miR-8, miR-12, miR-87, miR-190, miR-193 miR-263a, miR-275, miR-276, miR-276b, miR-279, miR-281 miR-282, miR-305, miR-315, miR-317, miR-750, miR-965 miR-993, miR-1000, miR-276a*, miR-281-2*, miR-8* miR-252b, miR-13a, miR-981, miR-bantam, miR-2001 miR-2c, miR-8, miR-12, miR-87, miR-190, miR-193 miR-263a, miR-275, miR-276, miR-276b, miR-279, miR-281 miR-282, miR-305, miR-315, miR-317, miR-750, miR-965 miR-993, miR-1000, miR-276a* , miR-281-2*, miR-8* miR-252b, miR-278, miR-981, miR-bantam, miR-2001

miR-S1, miR-S2 miR-S3, miR-S4 miR-S5, miR-S6 miR-S7, miR-S8 miR-S9, miR-S10 miR-S11, miR-S12 miR-S13, miR-S14 miR-S15

miR-S1, miR-S2 miR-S3, miR-S4 miR-S5, miR-S6 miR-S7, miR-S8 miR-S9, miR-S11 miR-S12, miR-S13 miR-S14 in other animals. At different times after infection, different but overlapping sets of shrimp miRNAs were expressed (Table 1), indicating changes in host miRNA expression during virus infection.

\section{Host miRNAs involved in virus infection}

To characterize the host miRNAs involved in virus infection, the expression profiles of miRNAs of virus-free and WSSV-infected shrimp at various times after infection were compared. To assess the significance of the observed changes in miRNA counts between the two different libraries, the Audice-Claverie test, the Fisher exact test, and the Chi-squared $2 \times 2$ test were used, with a Bonferroni correction for multiple comparisons. A $\mathrm{p}$-value $<0.01$ indicated that differences in the miRNA counts were statistically significant. The results showed that the expression patterns of many miRNAs did not significantly change in response to the WSSV infection, but 31 miRNAs (total counts $\geq 200$ ) were differentially expressed by more than twofold with a statistical significance of $\mathrm{p}<0.01$ (Figure 2a and Table 2). Comparison with the expression patterns of miRNAs at $0 \mathrm{~h}$ after infection showed that 25 miRNAs were significantly up-regulated by more than twofold: miR-1, miR100, miR-133, miR-184, miR-190, miR-193, miR-252, miR-263a, miR-275, miR-276a*, miR-281-2*, miR-2a, miR-2b, miR-2c, miR-315, miR-317, miR-34, miR-7, miR-71, miR-8*, miR-87, miR-965, miR-993, miR-let7 and miR-S2. Six miRNAs (miR-279, miR-33, miR-79, miR-9, miR-S5 and miR-S12) were significantly downregulated by more than twofold.
To confirm the involvement of these miRNAs in WSSV infection, 18 of them were selected at random for Northern blots. These showed expression patterns similar to those found by sequencing (Figure 2b); however, a little inconsistency was shown in miR-133, miR-193 and miR-2c compared with the results of sequencing, possibly owing to the low sensitivity of digoxigenin (DIG)-labeled oligodeoxynucleotide probes or for other unknown reasons.

\section{Pathways mediated by miRNAs}

To facilitate the prediction of the miRNA target gene, shrimp ESTs (162,926 EST reads) were assembled. The results showed that the assembled ESTs could be used for the miRNA target gene prediction. To reveal the interactions between the host miRNAs and virus genes, a total of 232 3' untranslated regions (UTRs) from the WSSV genome were used for target gene prediction. Analyses with the TargetScan and miRanda algorithms revealed the targets of 17 of the miRNAs that responded to virus infection (Table 3). The target genes were related to immune responses, gene expression regulation, signal transduction and metabolism. Some miRNAs, such as miR-34 and miRS12, could target 7-8 genes. One of the predicted viral target genes of miR-7 was wsv477, an early gene that might have a key role in DNA replication and virus proliferation [33]. The miR-7 might act as a regulator of components of the immune system to inhibit virus replication through their direct interaction with viral mRNA. However, fewer viral genes than host genes were targeted by host miRNAs (Table 3), suggesting that the short typical length of viral 3' UTRs evolved to minimize the effects of the host miRNAs. 
a

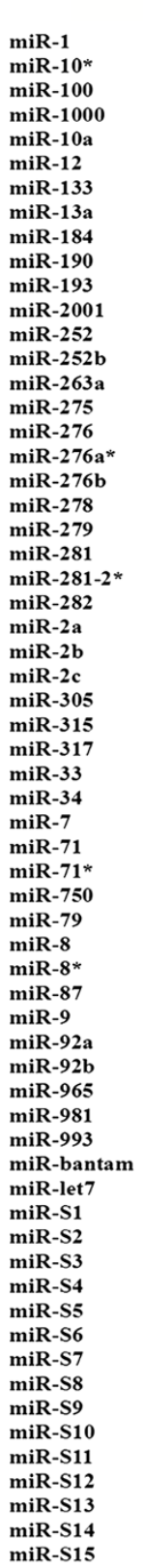

$\begin{array}{lllll}0 & 6 & 24 & 48 & \mathrm{~h}\end{array}$
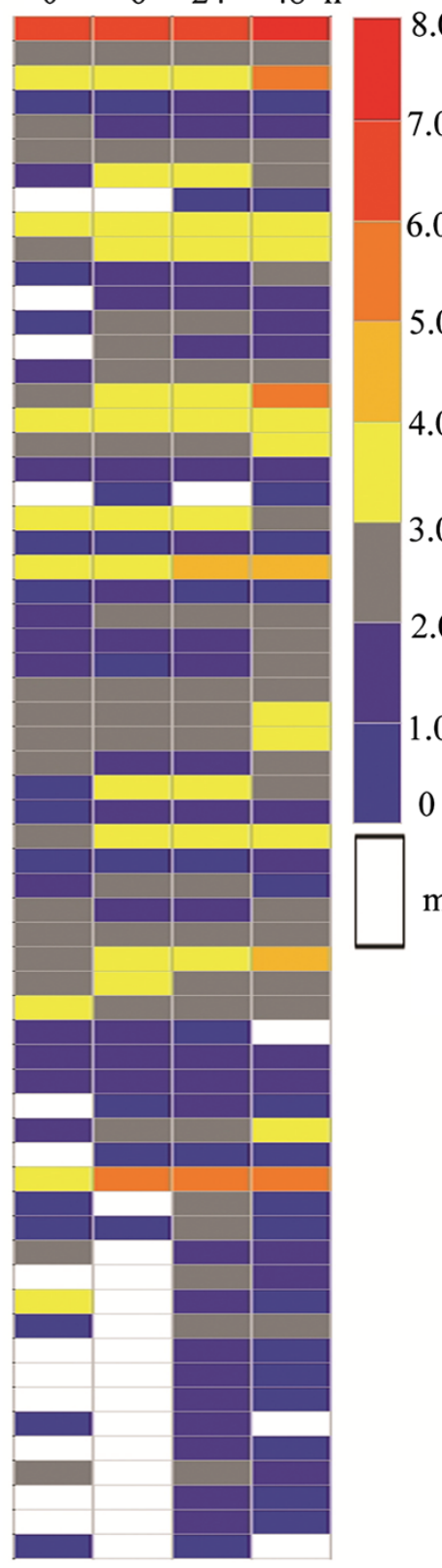

8.0

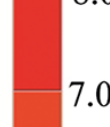

7.0

6.0

5.0

4.0

3.0

2.0

.0

miR-71

miR-87

miR-8*

miR-965

miR-let7

$\operatorname{miR}-279$

miR-33

$\operatorname{miR}-79$

miR-S5

miR-S12

U6

\section{$\begin{array}{llll}0 & 6 & 24 & 48 \mathrm{~h}\end{array}$}
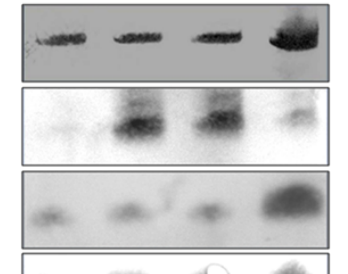

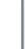
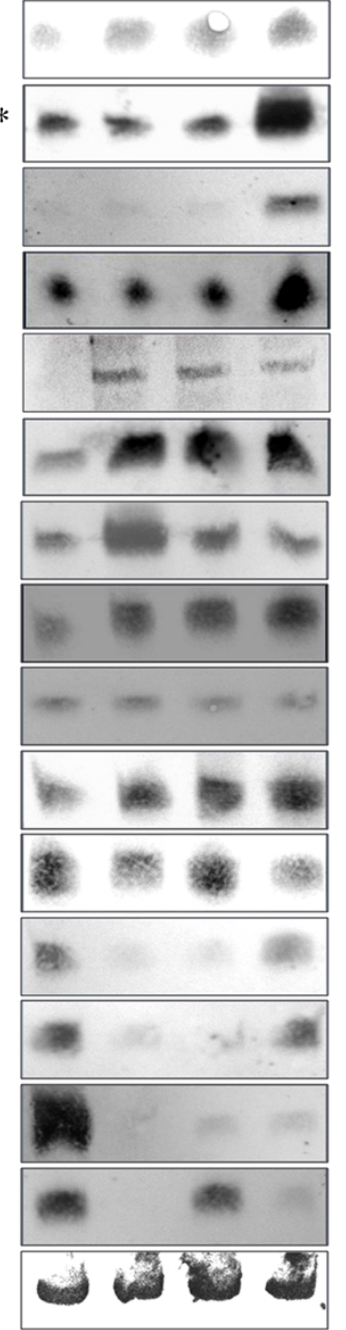

Figure 2 Expression of shrimp miRNAs in response to viral infection. (a) The miRNA expression profiles of WSSV-challenged shrimp at different time post-infection $(0,6,24$ and $48 \mathrm{~h}$ ). The numbers on the right indicated the log 10 of the number of copies of miRNAs. Miss, no copy detected. (b) Northern blots of selected shrimp miRNAs. Total RNA extracted from the lymphoid organs of the virus-free and WSSV-infected shrimp at different time post-infection $(0,6,24$ and $48 \mathrm{~h}$ ) were blotted with DIG-labeled oligodeoxynucleotide probes. The probes were shown at the left. U6 was used as a loading control.

To get an overview of the pathways mediated by host miRNAs, the target genes of miRNAs predicted by TargetScan were selected for gene ontology (GO) analysis. The results indicated that most of the target genes are involved in host immunity, including the small GTPase-mediated signaling transduction pathway, autophagy, phagocytosis, apoptosis, the Toll-like receptor signal pathway, antimicrobial humoral response, endocytosis, RNA interference (RNAi), response to viruses, virus-host interactions and regulation of innate immune response (Figure 3a). Moreover, a set of phagocytosis-related genes, such as myosin, actin, Arp2-Arp3 (Arp2/3), the serine/threonine kinase 
Table 2 Expression patterns of up-regulated or downregulated shrimp miRNAs after WSSV infection

\begin{tabular}{|c|c|c|c|c|}
\hline \multirow[t]{2}{*}{ MiRNA name } & \multirow{2}{*}{$\begin{array}{c}\text { Counts of } \\
0 \mathrm{hpi}\end{array}$} & \multicolumn{3}{|c|}{ Ratio of counts to $0 \mathrm{hpi}$ at } \\
\hline & & $6 \mathrm{hpi}$ & $24 \mathrm{hpi}$ & $48 \mathrm{hpi}$ \\
\hline \multicolumn{5}{|c|}{ up-regulation with $\geq 2$ fold } \\
\hline miR-1 & 1292519 & 1.53 & 1.21 & 2.74 \\
\hline miR-2a & 97 & 2.85 & 2.42 & 3.62 \\
\hline $\operatorname{miR}-2 b$ & 37 & 0.86 & 0.7 & 5.1 \\
\hline miR-2c & 15 & 0.56 & 0.73 & 23.46 \\
\hline miR-7 & 6 & 15.16 & 16 & 14.16 \\
\hline miR-34 & 9 & 174.55 & 120.11 & 18.77 \\
\hline miR-71 & 590 & 2.85 & 2.19 & 9.03 \\
\hline miR-87 & 229 & 5.23 & 2.32 & 2.96 \\
\hline miR-100 & 5550 & 0.8 & 0.69 & 20.89 \\
\hline miR-133 & 27 & 46.89 & 44.52 & 16.63 \\
\hline miR-184 & 1082 & 8.16 & 6.4 & 4.51 \\
\hline miR-190 & 389 & 3.67 & 2.79 & 6.69 \\
\hline miR-193 & 3 & 24.6 & 13.3 & 42.6 \\
\hline miR-252 & 7 & 36.29 & 34.14 & 8.14 \\
\hline miR-263a & 23 & 25.61 & 19.74 & 6.7 \\
\hline miR-275 & 364 & 18.74 & 10.28 & 87.04 \\
\hline miR-315 & 151 & 2.15 & 1.54 & 12.16 \\
\hline miR-317 & 121 & 3.79 & 2.63 & 9.02 \\
\hline miR-965 & 39 & 1.58 & 1.79 & 2.46 \\
\hline miR-993 & 69 & 7.19 & 4.1 & 16.07 \\
\hline miR-let7 & 6075 & 46.76 & 40.88 & 18.64 \\
\hline miR-281-2* & 1885 & 4.1 & 13.26 & 6.38 \\
\hline miR-8* & 787 & 6.42 & 3.82 & 13 \\
\hline miR-276a* & 459 & 1.46 & 1.09 & 14.15 \\
\hline miR-S2 & 6 & 1.16 & 37 & 0.83 \\
\hline \multicolumn{5}{|c|}{ Down-regulation with $\geq 2$ fold } \\
\hline miR-33 & 631 & 0.04 & 0.03 & 1.29 \\
\hline miR-79 & 895 & 0.04 & 0.03 & 0.19 \\
\hline miR-9 & 6175 & 0.05 & 0.05 & 0.15 \\
\hline $\operatorname{miR}-279$ & 2752 & 0.71 & 0.85 & 0.24 \\
\hline miR-S5 & 1063 & 0 & 0.06 & 0 \\
\hline miR-S12 & 376 & 0 & 0.67 & 0.03 \\
\hline
\end{tabular}

Array data for 31 host miRNAs with high-quality data (total counts $\geq 200$ ) at different time points after WSSV infection. hpi, hours post infection.

PAK and several members of the small G protein family, were enriched among the target genes of miR-79, suggesting that phagocytosis is an important immune strategy deployed by the host against virus infection [34]. Autophagy is an evolutionarily conserved mechanism of lysosomal degradation of unwanted cytoplasmic constituents, as well as of intracellular pathogens [35]. The GO analysis revealed that four genes targeted by miR-2 were classified into the autophagy subcategory, including the endogenous autophagosome marker protein microtubule-associated protein 1 light chain 3 (LC3), suggesting that miR-2 had important roles in the autophagy pathway.

The complementary binding between seed sequences of miRNAs and binding sites in target mRNAs might be conserved across species and might contribute to the functional conservation of miRNAs. Phylogenetic analysis, target gene prediction and pathway analysis showed that, among the 13 conserved miRNAs (miR-1, miR-100, miR-10a, miR-124, miR-125, miR-184, miR-33, miR-34, miR-7, miR-9, miR-92a, miR-92b and miR-let7), several highly conserved miRNAs (miR-1, miR-7 and miR-34) targeted the same or similar genes leading to the same pathways in shrimp, fruit fly and human (Figure 3b). This indicated that the beneficial miRNAs might be conserved during evolution because they aid survival.

\section{Discussion}

As is well known, virus infection can disturb and subvert the host cellular processions and functions at several levels, such as changes in the expression of cellular transcripts, including miRNAs, and effects on the cell cycle or apoptosis of virus-infected cells [36]. During virus-host interactions, cellular miRNAs, which are key regulators of gene expression, are crucial [14]. However, we have not yet achieved a comprehensive view of the gene expression regulation mediated by miRNAs during virus-host interactions in marine invertebrates. In this study, an invertebrate shrimp was challenged by the DNA virus WSSV so as to characterize the host miRNAs involved in the response to virus infection. The results showed that 31 host miRNAs are involved in virus-host interactions, most of which are concerned with host immune responses. Our study provides the first large-scale characterization of marine invertebrate miRNAs and the pathways mediated by them in response to virus challenge.

Similar phenomena have been reported in mammals, the miRNA profiles of which are reshaped by hepatitis $C$ virus (HCV), human immunodeficiency virus-1, human cytomegalovirus and Epstein-Barr virus [16,37-39]. The host miRNAs might be associated with the regulation of host immune systems or viral life cycles. RNAi knockdowns of Drosha and Dicer, two crucial proteins in animal miRNA biogenesis, resulted in a decrease in mature host miRNAs, which led to increased sensitivity of host to virus infection $[15,40]$. Some host miRNAs might thus represent antiviral miRNAs. When some putative antiviral miRNAs were blocked by locked nucleic acid-modified antisense oligoribonucleotides, the hosts failed to inhibit viral replication [11,41]. In some cases, host miRNA expression might be promoted by viruses to reshape the host intracellular environment to benefit viral replication [42]. In our study, phylogenetic analysis showed that the miR-1, miR-7 and 
Table 3 Target genes of miRNAs predicted by TargetScan and miRanda algorithms

\begin{tabular}{|c|c|}
\hline miRNA & overlapping genes \\
\hline miR-1 & KRAB domain-containing zinc finger protein \\
\hline miR-12 & acyl-CoA binding domain containing 7 \\
\hline miR-133 & ubiquitin-conjugating enzyme E2 A \\
\hline miR-13a & O-methyltransferase; fibroinase; Mn superoxide dismutase; DNA-binding nuclear protein p8; peroxin-11 C \\
\hline miR-2001 & Scavenger receptor class B; glycoprotein 25 l; vp28 (WSSV) \\
\hline miR-263a & Dihydropteridine reductase; Tetraspanins-like protein \\
\hline miR-275 & Peritrophin $\mathrm{A}$; calcium and integrin binding protein $\mathrm{CIB}$ \\
\hline miR-276b & Vacuolar ATP synthase \\
\hline miR-278 & eukaryotic translation initiation factor 3 subunit \\
\hline miR-279 & ubiquitin protein ligase; Exoskeletal protein \\
\hline miR-281 & eukaryotic translation elongation factor 1 alpha (eEF-1a); elongation factor EF-1 alpha subunit \\
\hline miR-282 & ribose 5-phosphate isomerase A; small subunit ribosomal protein S6e \\
\hline miR-2a & Melanization interacting protein; Fortilin binding protein 1; O-methyltransferase \\
\hline miR-317 & $\begin{array}{l}\text { hfb2 protein; (R)-3-amino-2-methylpropionate-pyruvate transaminase; Eukaryotic initiation factor 1A; } \\
\text { maleylacetoacetate isomerase; hfb2 protein }\end{array}$ \\
\hline miR-34 & $\begin{array}{l}\text { solute carrier family } 37 \text { (glycerol-3-phosphate transporter), member 2; Rpl6, NV12167; ribosomal protein } \\
\text { L6; K02934 large subunit ribosomal protein L6e; ubiquitin protein ligase; K10573 ubiquitin-conjugating } \\
\text { enzyme E2 A; Nuclear autoantigenic sperm protein; similar to elongase, putative; E3 ubiquitin ligase }\end{array}$ \\
\hline miR-7 & eukaryotic translation initiation factor 4A2; translation initiation factor elF-4A; wsv477 (WSSV) \\
\hline miR-71 & actin 1; microsomal signal peptidase; signal peptidase complex subunit 3 \\
\hline miR-8* & hfb2 protein \\
\hline miR-87 & alanine-glyoxylate transaminase \\
\hline miR-92a & Eukaryotic initiation factor $1 \mathrm{~A}$; maleylacetoacetate isomerase \\
\hline miR-let7 & rab11; T-complex protein 1 subunit gamma \\
\hline miR-S1 & Astacin \\
\hline miR-S10 & $\begin{array}{l}\text { Rnps1 protein; RNA-binding protein with serine-rich domain 1; nuclear distribution protein NUDC; } \\
\text { isopentenyl-diphosphate delta isomerase } 1\end{array}$ \\
\hline miR-S12 & $\begin{array}{l}\text { ribosomal protein L5; Glucosyl/glucuronosyl transferase (Fragment); myosin heavy chain; Cationic } \\
\text { trypsin-3 precursor Pretrypsinogen III; trypsin; eukaryotic translation initiation factor 2B; ribosomal } \\
\text { protein L13A; microsomal glutathione S-transferase; glutathione S-transferase; methionyl aminopeptidase }\end{array}$ \\
\hline miR-S13 & similar to ribosomal protein L28; K02903 large subunit ribosomal protein L28e \\
\hline miR-S14 & putative beta-NAC-like protein; phosphatidylserine receptor \\
\hline miR-S3 & acireductone dioxygenase; ubiquitin C-terminal hydrolase; Duplex-specific nuclease \\
\hline miR-S5 & phosphatidylserine receptor; cystatin B \\
\hline miR-S6 & Glucosyl/glucuronosyl transferase \\
\hline miR-S9 & 3-hydroxyisobutyryl-CoA hydrolase \\
\hline
\end{tabular}

miR-34 are highly conserved in shrimp, fruit fly and human and function in similar pathways. Our analyses predicted that miR-7, one of the miRNAs highly conserved between invertebrates and vertebrates, could target the mitogen-activated protein kinases (MAPKs), a situation identical to that in humans [43-45]. Recent studies revealed that MAPKs were activated by invading $\mathrm{HCV}$, the orthopoxvirus vaccinia virus and visna virus, which aided viral replication [43-45]. Our analysis indicated that the WSSV early gene wsv477 was also targeted by host miR-7, suggesting that host might inhibit virus infection by targeting viral transcripts with host miRNAs. It could thus be inferred that the functions of the conserved miRNAs have been preserved in animals during evolution. Because of the long evolutionary time since the divergence of shrimps and humans, studies on invertebrates would greatly benefit from even limited knowledge about shrimp virus-host interactions.

In our study, Solexa high-throughput deep sequencing was used to reveal the miRNAs involved in virus-host interactions. A total of 63 miRNAs were obtained, but no viral miRNA was revealed. This might be because of 


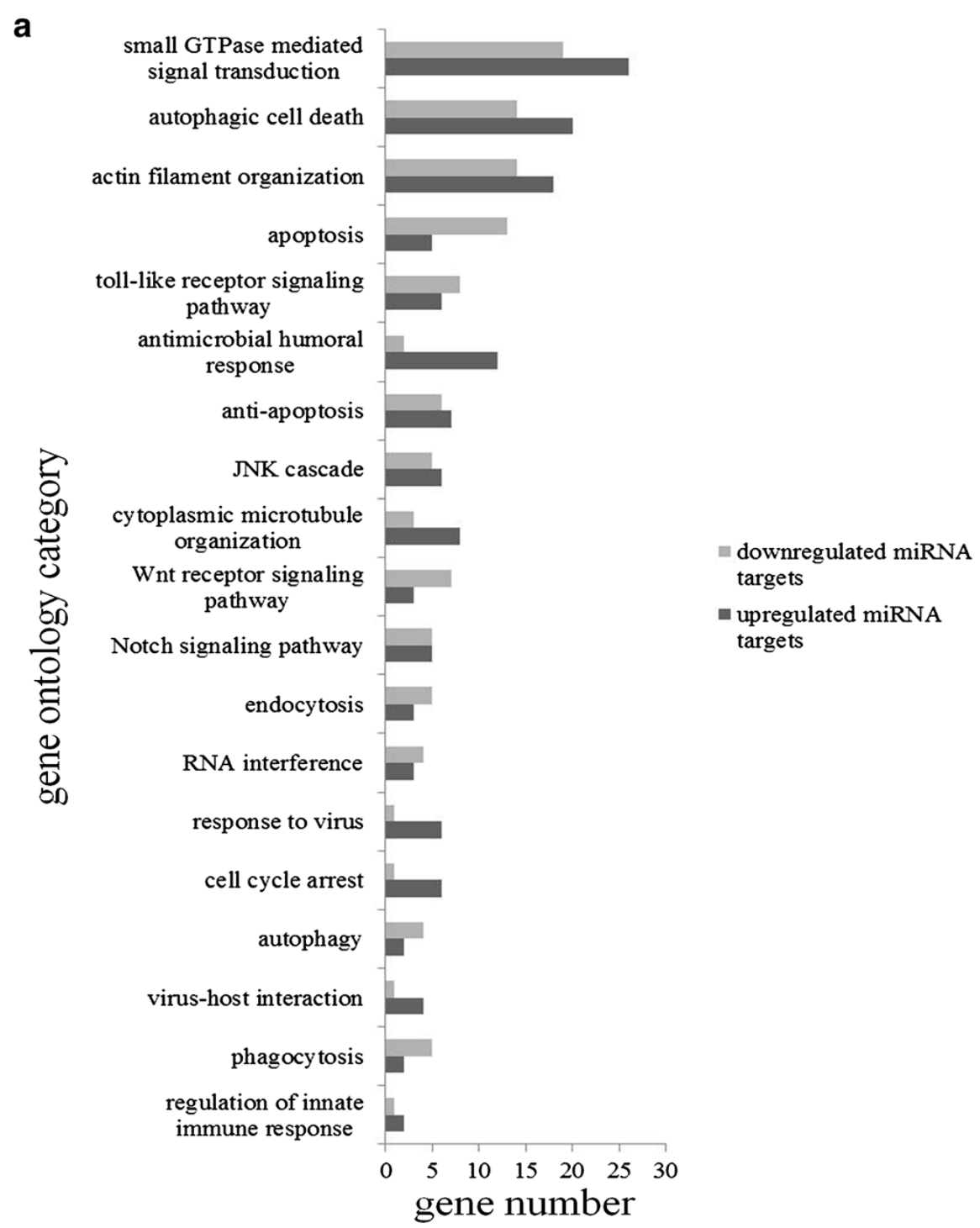

b

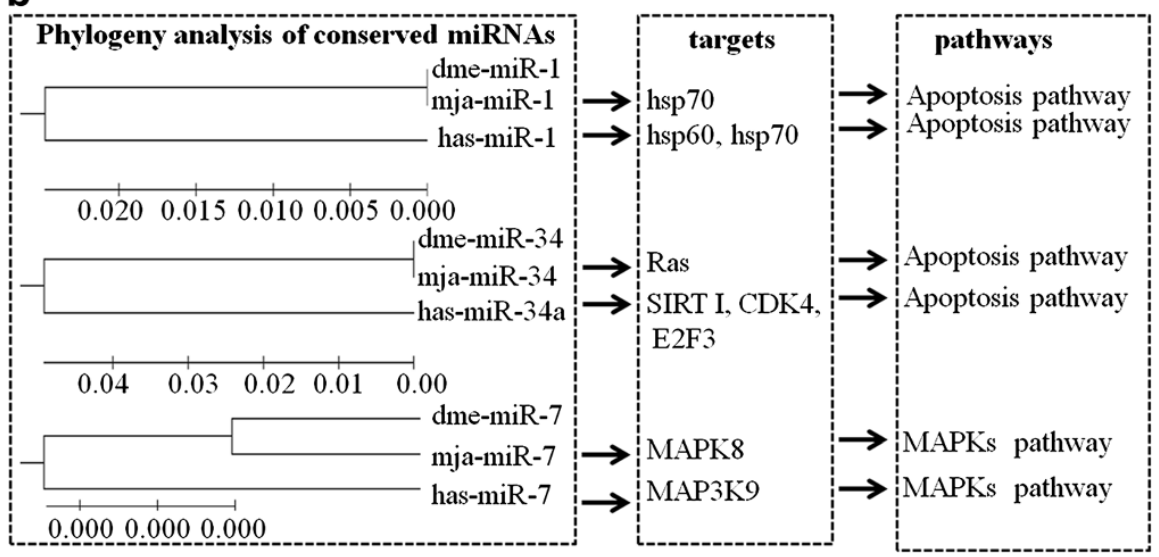

Figure 3 Pathways mediated by miRNAs. (a) GO analysis of genes targeted by miRNAs involved in virus-host interactions. Numbers indicated the total numbers of genes targeted by down- or up-regulated miRNAs in each putative functional category. (b) Pathways mediated by the miRNAs conserved with other animals. mja: Marsupenaeus japonicus; dme: Drosophila melanogaster; hsa: Homo sapiens. 
the small amounts of viral miRNAs. To characterize the viral miRNAs, an miRNA microarray could be used in the further studies.

\section{Conclusions}

Our study provides the first large-scale characterization of marine invertebrate miRNAs in response to virus infection. The results showed that a total of 63 miRNAs of shrimp were obtained, 31 out of which were differentially expressed in response to virus infection. Among the differentially expressed miRNAs found, miR-1, miR-7 and miR-34 are highly conserved and mediate similar pathways, suggesting that some beneficial miRNAs have been preserved in animals during evolution. Invertebrates could therefore be good candidates for increasing our still limited knowledge about virus-host interactions because of their long evolutionary distance from vertebrates. Our study could help to reveal the molecular events of virus-host interactions mediated by miRNAs and their evolution in animals.

\section{Materials and methods}

\section{Shrimp culture and WSSV infection}

M. japonicus shrimp (10-15 g body weight) were cultured in groups of 20 individuals in each tank with artificial seawater and aeration. Before the experiments, the shrimp were maintained temporarily for 2-3 days and three shrimp were randomly selected for WSSV detection with WSSV-specific primers to ensure that the shrimp were virus-free. Then the virus-free shrimp were infected with WSSV at $10^{4}$ virions per $\mathrm{ml}$ by intramuscular injection using a syringe with a 29-gauge needle [46]. After WSSV challenge, the lymphoid organs of five individuals were collected at various times after infection $(0$, 6, 24 and $48 \mathrm{~h}$ ) and immediately stored in liquid nitrogen for later use. Shrimp assays were conducted in accordance with COPE (the Committee on Publication Ethics).

\section{Sequencing of small RNAs}

Total RNAs were isolated from the lymphoid organs of the virus-free and WSSV-infected shrimp at different times after infection using a mirVana miRNA Isolation Kit (Ambion, Austin, TX) according to the manufacturer's instructions. The quantity and purity of total RNAs were monitored using a NanoDrop ND-1000 spectrophotometer (Nano Drop, DE) at a 260/280 ratio > 2.0. The integrity of total RNAs was analyzed using an Agilent 2100 Bioanalyzer system and an RNA 6000 Nano LabChip Kit (Agilent, CA) with an RNA integrity number (RIN) > 8.0. About $200 \mu \mathrm{g}$ of total RNA was separated on a denaturing $15 \%$ polyacrylamide gel. The small RNAs (16-30 nt) were excised, quantified and precipitated with ethanol. After dephosphorylation by alkaline phosphatase, the purified small RNAs were ligated sequentially to RNA adapters
(5'-ACAGGUUCAGAGUUCUACAGUCCGACGAUC-3' and 5'-UCGUAUGCCGUC UUCUGCUUG-3'). Reverse transcription and PCR amplification were performed after ligation. The resulting products were sequenced on the Genome Analyzer GA-I (Illumina, San Diego, CA) according to the manufacturer's recommended protocol.

\section{Small RNA sequence analysis}

Illumina's Genome Analyzer Pipeline software and the ACGT V3.1 program developed by LC Sciences (Houston, TX) were used for small RNA sequence analysis. The following sequences were removed: (1) sequences of the vector and adaptor, (2) low-quality sequences, (3) low-copy sequences (counts $<3$ ), (4) sequences containing more than $80 \% \mathrm{~A}, \mathrm{C}, \mathrm{G}$, or $\mathrm{T}$, (5) sequences containing only A and $\mathrm{C}$ or only $\mathrm{G}$ and $\mathrm{T}$, (6) sequences shorter than $16 \mathrm{nt}$ and longer than $26 \mathrm{nt}$, (7) sequences containing 10 repeats of any dimer, 6 repeats of any trimer, or 5 repeats of any tetramer, (8) sequences matching mRNAs, rRNA, tRNA, snRNA, snoRNA. After these sequences were removed, all the remaining high-quality sequences were used for miRNA identification. To identify conserved miRNAs that were homologous with those of other species, all highquality sequences were mapped to known mature and precursor arthropod miRNAs in miRBase 15.0 with an Evalue similarity cutoff of $1 \mathrm{e}-10$, and the pre-miRNAs were further mapped to the ESTs of the shrimp Litopenaeus vannamei from GenBank owing to the lack of the Marsupenaeus japonicus genome. To characterize novel miRNA candidates in shrimp, the remaining high-quality sequences with no homologs in miRBase 15.0 were analyzed by a BLASTN search against the shrimp EST database in the National Center for Biotechnology Information [47], allowing one or two mismatches between each pair of sequences. Hairpin RNA structures were predicted from the $65 \mathrm{nt}$ sequences adjacent to the mapped ESTs in either direction by the MFOLD program using default parameters [48].

\section{Northern blotting}

Total RNA was extracted from the lymphoid organs of the virus-free and WSSV-infected shrimp at different times post-infection $(0,6,24$ and $48 \mathrm{~h})$ and quantified using a spectrophotometer (NanoDrop, Wilmington, USA). Then, $30 \mu \mathrm{g}$ of total RNA was separated on a denaturing $15 \%$ polyacrylamide gel containing $8 \mathrm{M}$ urea. The RNA was transferred to Hybond-N + membranes (Amersham Biosciences, Buckinghamshire, UK). After ultraviolet crosslinking $(120 \mathrm{~mJ}, 30 \mathrm{~s})$, the membrane was pre-hybridized in DIG Easy Hyb granules buffer (Roche, Basel, Switzerland) for $0.5 \mathrm{~h}$, and this was followed by hybridization with a DIG-labeled DNA probe complementary to a specific miRNA sequence for $20 \mathrm{~h}$. The DIG labeling and detection were performed following the manual of DIG High Prime DNA Labeling and Detection Starter Kit II (Roche). 


\section{Shrimp EST assembly and 3' UTR extraction}

Because of the lack of a shrimp genome sequence, the EST database containing 162,926 EST reads of the shrimp Litopenaeus vannamei from GenBank was used for the prediction of miRNA target genes [47]. However, most of the EST reads were too short ( $\leq 500 \mathrm{bp}$ ) to include information on 3' UTR sequences, which were the regions usually targeted by miRNAs. Therefore, the EST sequences were assembled using the CAP3 assembly program into a total of 31,831 non-redundant sequences comprising contigs and singlets $[49,50]$. According to the highest BLASTX and/or BLASTN hits, the most likely open reading frames were annotated, and their corresponding 3' UTRs were determined through to the polyadenylation signal. A poly(A) signal was taken as a sequence of ATTAAA or AATAAA located 10-35 nt from either the poly $(\mathrm{A})$ tail or the end of the sequence. A poly(A) tail was taken as a run of at least six As at the end of a sequence. Incomplete 3' UTRs were removed from further analysis. To characterize the interactions between the host miRNAs and WSSV genes, a total of 232 3' UTRs from the WSSV genome sequence [GenBank: NC_003225] were extracted as described above.

\section{Prediction of genes targeted by miRNAs}

To predict the genes targeted by miRNAs, two computational target prediction algorithms, TargetScan 5.1 and miRanda, were used [51,52]. The data-sets used were the assembled EST sequences and the 3' UTRs of WSSV. TargetScan was used to search for miRNA seed matches (nucleotides 2-8 from the $5^{\prime}$ end of miRNA) in the $3^{\prime}$ UTR sequences. miRanda was used to match the entire miRNA sequences. The miRanda parameters were set as free energy $<-20 \mathrm{kcal} / \mathrm{mol}$ and score $>50$. Finally, the results predicted by the two algorithms were combined and the overlaps were calculated.

\section{Gene ontology (GO) analysis}

The coding sequences of the shrimp ESTs were extracted and used as queries to search the protein sequences collected by the GO database with the blast $\mathrm{E}$ value $<1 \mathrm{e}-5$ [53]. The best hit GO IDs were assigned to the shrimp EST sequences. The hypergeometric test statistic was then used to obtain the over-representation of particular functions or categories in the data of miRNA targets predicted by TargetScan 5.1 as compared with all the EST data. The $\mathrm{P}$ values were corrected by false discovery rate (FDR).

\section{Additional files}

Additional file 1: Small RNA reads sequenced by Solexa technology from WSSV-infected shrimp at different time post-infection $(0,6,24$, and $48 \mathrm{~h}$ ). Statistical analyses were based on the counts and percentages of the raw, mappable sequences and unique miRNA reads from virus-free WSSV-infected shrimps.
Additional file 2: The shrimp miRNAs that were conserved in other animals. The 48 miRNAs conserved in other animals were classified into 43 distinct families

Additional file 3: The mapped EST sequences of shrimp-specific miRNAs. The mature sequence of each shrimp-specific miRNA was indicated as a different case in the fasta EST sequence.

\section{Abbreviations}

MiRNAs: MicroRNAs; WSSV: White spot syndrome virus; GO: Gene ontology; EST: Expressed sequence tag; NCBI: The National Center for Biotechnology Information; DIG: Digoxigenin; UTRs: Untranslated regions; FDR: False discovery rate; PAK: Serine/threonine p21-activated kinase; LC3: Microtubule-associated protein 1 light chain 3; HCV: Hepatitis C virus; HIV-1: Human immunodeficiency virus-1; HCMV: Human cytomegalovirus; EBV: Epstein-Barr virus; MAPKs: Mitogen-activated protein kinases; Mja: Marsupenaeus japonicus; Dme: Drosophila melanogaster; Hsa: Homo sapiens; RIN: RNA integrity number; Hpi: Hours post infection.

\section{Competing interests}

The authors declare that they have no competing interests.

\section{Acknowledgements}

This work was financially supported by National Natural Science Foundation of China (30830084) and Hi-Tech Research and Development Program of China (863 program of China) (2010AA09Z403, 2011AA10A216).

\section{Authors' contributions}

TZH performed the experiments, gathered and analyzed the data and drafted the manuscript. DDX performed some of the experiments and aided in writing the manuscript. XBZ designed the investigation, acquired and analyzed the data, and finished the final version of the manuscript. All authors carefully checked and approved this version of the manuscript.

Received: 30 March 2012 Accepted: 30 April 2012

Published: 30 April 2012

\section{References}

1. Carrington JC, Ambros V: Role of microRNAs in plant and animal development. Science 2003, 301:336-338.

2. Bernstein E, Caudy AA, Hammond SM, Hannon GJ: Role for a bidentate ribonuclease in the initiation step of RNA interference. Nature 2001, 409:363-366.

3. Bartel DP: MicroRNAs: genomics, biogenesis, mechanism, and function. Cell 2004, 116:281-297.

4. Meister G, Tuschl T: Mechanisms of gene silencing by double-stranded RNA. Nature 2004, 431:343-349.

5. Schwarz DS, Hutvagner G, Du T, Xu Z, Aronin N, Zamore PD: Asymmetry in the assembly of the RNAi enzyme complex. Cell 2003, 115:199-208.

6. Bagga S, Bracht J, Hunter S, Massirer K, Holtz J, Eachus R, Pasquinelli AE: Regulation by let-7 and lin-4 miRNAs results in target mRNA degradation. Cell 2005, 122:553-563.

7. Baek D, Villén J, Shin C, Camargo FD, Gygi SP, Bartel DP: The impact of microRNAs on protein output. Nature 2008, 455:64-71.

8. Lim LP, Lau NC, Garrett-Engele P, Grimson A, Schelter JM, Castle J, Bartel DP, Linsley PS, Johnson JM: Microarray analysis shows that some microRNAs downregulate large numbers of target mRNAs. Nature 2005, 433:769-773.

9. Ambros V: The functions of animal microRNAs. Nature 2004, 431:350-355.

10. Teleman AA, Maitra S, Cohen SM: Drosophila lacking microRNA miR- 278 are defective in energy homeostasis. Genes Dev 2006, 20:417-422.

11. Pedersen IM, Cheng G, Wieland S, Volinia S, Croce CM, Chisari FV, David M: Interferon modulation of cellular microRNAs as an antiviral mechanism. Nature 2007, 499:919-922.

12. Huang J, Wang F, Argyris E, Chen K, Liang Z, Tian H, Huang W, Squires K, Verlinghieri G, Zhang H: Cellular micro-RNAs contribute to HIV-1 latency in resting primary CD4+ T lymphocytes. Nat Med 2007, 13:1241-1247.

13. Lecellier CH, Dunoyer P, Arar K, Lehmann-Che J, Eyquem S, Himber C, Saib A, Voinnet O: A cellular microRNA mediates antiviral defense in human cells. Science 2005, 308:557-560. 
14. Umbach $J$, Cullen BR: The role of RNAi and microRNAs in animal virus replication and antiviral immunity. Genes Dev 2009, 23:1151-1164.

15. Triboulet R, Mari B, Lin YL, Chable-Bessia C, Bennasser $Y$, Lebrigand $K$, Cardinaud B, Maurin T, Barbry P, Baillat V, Reynes J, Corbeau P, Jeang KT, Benkirane M: Suppression of microRNA-silencing pathway by HIV-1 during virus replication. Science 2007, 315:1579-1582.

16. Wang FZ, Weber F, Croce C, Liu CG, Liao X, Pellett PE: Human cytomegalovirus infection alters the expression of cellular microRNA species that affect its replication. J Virol 2008, 82:9065-9074.

17. Fullaondo A, Lee SY: Regulation of Drosophila-virus interaction. Dev Comp Immunol 2012, 36:262-266.

18. Lee RC, Feinbaum RL, Ambros V: The C. elegans heterochronic gene lin-4 encodes small RNAs with antisense complementarity to lin-14. Cell 1993, 75:843-854.

19. Reinhart BJ, Slack FJ, Basson M, Pasquinelli AE, Bettinger JC, Rougvie AE, Horvitz HR, Ruvkun G: The 21-nucleotide let-7 RNA regulates developmental timing in Caenorhabditis elegans. Nature 2000, 403:901-906.

20. Griffiths-Jones S, Grocock RJ, van Dongen S, Bateman A, Enright AJ: miRBase: microRNA sequences, targets and gene nomenclature. Nucleic Acids Res 2006, 34:D140-144.

21. Grosshans H, Slack FJ: Micro-RNAs: small is plentiful. J Cell Biol 2002, 156:17-21.

22. Bartel DP: MicroRNAs: target recognition and regulatory functions. Cell 2009, 136:215-233.

23. Lim LP, Lau NC, Weinstein EG, Abdelhakim A, Yekta S, Rhoades MW, Burge CB, Bartel DP: The microRNAs of Caenorhabditis elegans. Genes Dev 2003, 17:991-1008.

24. Lewis BP, Burge CB, Bartel DP: Conserved seed pairing, often flanked by adenosines, indicates that thousands of human genes are microRNA targets. Cell 2005, 120:15-20.

25. Enright AJ, John B, Gaul U, Tuschl T, Sander C, Marks DS: MicroRNA targets in Drosophila. Genome Biol 2003, 5(1):R1.

26. Wang $X$, Zhang J, Li F, Gu J, He T, Zhang X, Li Y: MicroRNA identification based on sequence and structure alignment. Bioinformatics 2005, 21:3610-3614

27. Grad Y, Aach J, Hayes GD, Reinhart BJ, Church GM, Ruvkun G, Kim J: Computational and experimental identification of $C$. elegans microRNAs. Mol Cell 2003, 11:1253-1263.

28. Ambros $V$, Lee RC: Identification of microRNAs and other tiny noncoding RNAs by cDNA cloning. Methods Mol Biol 2004, 265:131-158.

29. Chen PY, Manninga H, Slanchev K, Chien M, Russo JJ, Ju J, Sheridan R, John B, Marks DS, Gaidatzis D, Sander C, Zavolan M, Tuschl T: The developmental miRNA profiles of zebrafish as determined by small RNA cloning. Genes Dev 2005, 19:1288-1293.

30. Schafer A, Cai X, Bilello JP, Desrosiers RC, Cullen BR: Cloning and analysis of microRNAs encoded by the primate gamma-herpesvirus rhesus monkey rhadinovirus. Virology 2007, 364:21-27.

31. Schreiber AW, Shi BJ, Huang CY, Langridge $P$, Baumann U: Discovery of barley miRNAs through deep sequencing of short reads. BMC Genomics 2011, 12:129.

32. Chang PS, Chen HC, Wand YC: Detection of white spot syndrome associated baculovirus WSBV in experimentally infected wild shrimps crabs and lobsters by in situ hybridization. Aquaculture 1998, 164:23-43.

33. Han F, Xu J, Zhang X: Characterization of an early gene (wsv477) from shrimp white spot syndrome virus (WSSV). Virus Genes 2007, 34(2):193-198.

34. Aderem A, Underhill DM: Mechanisms of phagocytosis in macrophages. Annu Rev Immunol 1999, 17:593-623.

35. Klionsky DJ, Emr SD: Autophagy as a regulated pathway of cellular degradation. Science 2000, 290:1717-1721.

36. Di Bartolo DL, Cannon M, Liu YF, Renne R, Chadburn A, Boshoff C, Cesarman E: KSHV LANA inhibits TGF-b signaling through epigenetic silencing of the TGF-b type II receptor. Blood 2008, 111:4731-4740.

37. Varnholt H, Drebber U, Schulze F, Wedemeyer I, Schirmacher P, Dienes HP, Odenthal M: MicroRNA gene expression profile of hepatitis $C$ virusassociated hepatocellular carcinoma. Hepatology 2008, 47:1223-1232.

38. Houzet L, Yeung ML, de Lame V, Desai D, Smith SM, Jeang KT: MicroRNA profile changes in human immunodeficiency virus type 1 (HIV-1) seropositive individuals. Retrovirology 2008, 5:118.

39. Cameron JE, Fewell C, Yin Q, McBride J, Wang X, Lin Z, Flemington EK: Epstein-Barr virus growth/latency III program alters cellular microRNA expression. Virology 2008, 382(2):257-266.
40. Matskevich AA, Moelling K: Dicer is involved in protection against influenza A virus infection. J Gen Virol 2007, 88:2627-2635.

41. Wang X, Ye L, Hou W, Zhou Y, Wang YJ, Metzger DS, Ho WZ: Cellular microRNA expression correlates with susceptibility of monocytes/ macrophages to HIV-1 infection. Blood 2009, 113:671-674.

42. Jopling CL, Yi MK, Lancaster AM, Lemon SM, Sarnow P: Modulation of hepatitis $C$ virus RNA abundance by a liver-specific micro- RNA. Science 2005, 309:1577-1581.

43. Hayashi J, Aoki H, Kajino K, Moriyama M, Arakawa Y, Hino O: Hepatitis C virus core protein activates the MAPK/ERK cascade synergistically with tumor promoter TPA, but not with epidermal growth factor or transforming growth factor alpha. Hepatology 2000, 32(5):958-961.

44. Andrade AA, Silva PN, Pereira AC, De Sousa LP, Ferreira PC, Gazzinelli RT, Kroon EG, Ropert C, Bonjardim CA: The vaccinia virus-stimulated mitogenactivated protein kinase (MAPK) pathway is required for virus multiplication. Biochem J 2004, 15; 381(Pt 2):437-446.

45. Barber SA, Bruett L, Douglass BR, Herbst DS, Zink MC, Clements JE: Visna virus-induced activation of MAPK is required for virus replication and correlates with virus-induced neuropathology. J Virol 2002, 76(2):817-828.

46. Wu WL, Wang $L$, Zhang XB: Identification of white spot syndrome virus (WSSV) envelope proteins involved in shrimp infection. Virology 2005, 332:578-583.

47. NCBI [http://www.ncbi.nlm.nih.gov/]

48. MFOLD program (version 2.38) [http://mfold.rna.albany. edu/?q=mfold/ RNA-Folding-Form]

49. CAP3 Sequence Assembly Program [http://pbil.univ-lyon1.fr/cap3.php]

50. Huang X, Madan A: CAP3: A DNA sequence assembly program. Genome Res 1999, 9(9):868-877.

51. TargetScan 5.1 [http://www.targetscan.org]

52. miRanda [http://www.microrna.org/]

53. Gene Ontology database [http://www.geneontology.org]

doi:10.1186/1471-2164-13-159

Cite this article as: Huang et al:: Characterization of host microRNAs that respond to DNA virus infection in a crustacean. BMC Genomics 2012 13:159.

\section{Submit your next manuscript to BioMed Central and take full advantage of:}

- Convenient online submission

- Thorough peer review

- No space constraints or color figure charges

- Immediate publication on acceptance

- Inclusion in PubMed, CAS, Scopus and Google Scholar

- Research which is freely available for redistribution 